\title{
HIGH-FIDELITY SIMULATION — THE FIRST DCD-ECMO PROCEDURE IN POLAND
}

\author{
Mateusz Puslecki ${ }^{1,2}$, Marcin Ligowski ${ }^{1}$, Marek Dabrowski ${ }^{2,3}$, Maciej Sip ${ }^{2,3}$, Sebastian Stefaniak ${ }^{1}$, \\ Tomasz Klosiewicz ${ }^{2,3}$, Lukasz Gasiorowski ${ }^{3-5}$, Marek Karczewski ${ }^{2,6}$, Tomasz Malkiewicz ${ }^{7,8}$, \\ Malgorzata Ladzinska ${ }^{1}$, Marcin Zielinski ${ }^{2,8}$, Aleksander Pawlak ${ }^{8}$, Agata Dabrowska ${ }^{2,3}$, Piotr Ziemak ${ }^{4}$, \\ Bartlomiej Perek ${ }^{1}$, Marcin Misterski ${ }^{1}$, Slawomir Katarzynski ${ }^{1}$, Piotr Buczkowski ${ }^{1}$, Wojciech Telec ${ }^{2}$, \\ Ilona Kiel-Puslecka ${ }^{9}$, Michal Kiel ${ }^{10}$, Michael Czekajlo ${ }^{3,11,12}$, Marek Jemielity ${ }^{1}$ \\ ${ }^{1}$ Poznan University of Medical Sciences, Department of Cardiac Surgery and Transplantology, Clinical Hospital SKPP, Poznan, Poland \\ ${ }^{2}$ Poznan University of Medical Sciences, Department of Rescue and Disaster Medicine, Poznan, Poland \\ ${ }^{3}$ Polish Society of Medical Simulation, Poland \\ ${ }^{4}$ Poznan University of Medical Sciences, Center for Medical Simulation, Poznan, Poland \\ ${ }^{5}$ Poznan University of Medical Sciences, Department of Intensive Care and Pain Treatment, Poznan, Poland \\ ${ }^{6}$ Poznan University of Medical Sciences, Department of Transplantology, General, Vascular and Plastic Surgery, Poznan, Poland \\ ${ }^{7}$ Poznan University of Medical Sciences, Department of Anesthesiology and Intensive Care, Clinical Hospital H. Święcickiego, Poznan, Poland \\ ${ }^{8}$ Voivodeship Emergency Medical Services, Poznan, Poland \\ ${ }^{9}$ Poznan University of Medical Sciences, Department of Palliative Medicine, Poznan, Poland \\ ${ }^{10}$ ZF RTW, Częstochowa, Poland \\ ${ }^{11}$ Hunter Holmes McGuire VA Medical Center, Department of Surgery, Richmond, United States of America \\ ${ }^{12}$ Lublin Medical University, Lublin, Poland
}

Disaster Emerg Med J 2017; (2)1: 50-52

Dear Sir,

Although kidney transplantation has become an effective means of treating end-stage renal disease (ESRD), many patients die waiting for organ transplant. This problem is not an isolated phenomenon in Poland (13.6 donors per 1 million inhabitants) but in other European countries, despite the much greater number of organs transplanted each year (from 23.4 donors per 1 million inhabitants in the Czech Republic 40.2 per million in Spain), Poland continues to seek and create opportunities for the development program of organ procurement and transplantation. The alternative option is the inclusion donor after circulatory death (DCD) in the transplantation program, for which circulatory criteria are used to define death. The complicated procedure required to maintain the viability of organs for transplantation and dramatic time criteria in the absence of appropriate organizational capabilities have caused that transplantation from DCD donors is not yet widespread in Poland [1-3].
We present the procedural preparation for the Poland's first case of organ (kidney) transplantation from a circulatory death donor in which perfusion was supported by Extracorporeal Membrane Oxygenation (ECMO). The procedure is a component of the "ECMO for Wielkopolska" program established to serve 3.5 million inhabitants of the Wielkopolska region in Poland. Because this organizational model is complex and expensive, we used advanced

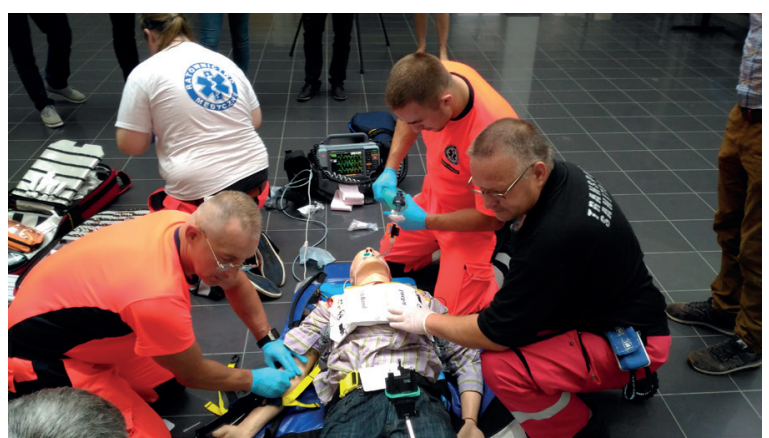

FIGURE 1. SCA in public-ALS on MCC 


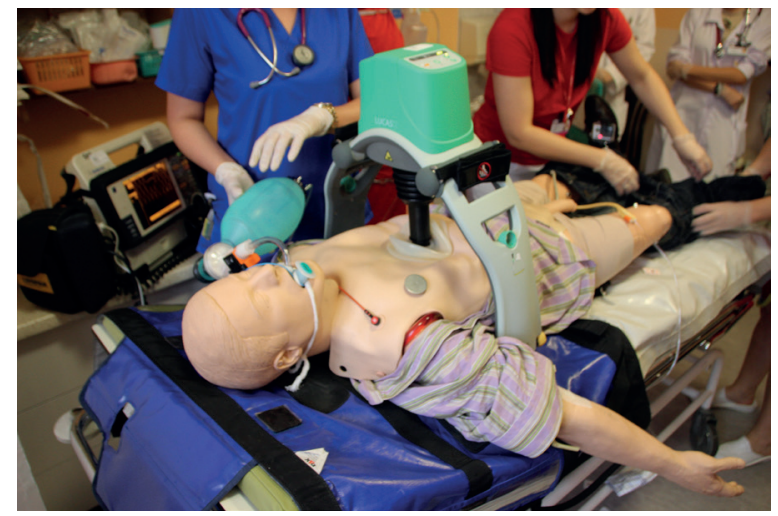

FIGURE 2. ALS on MCC in ED

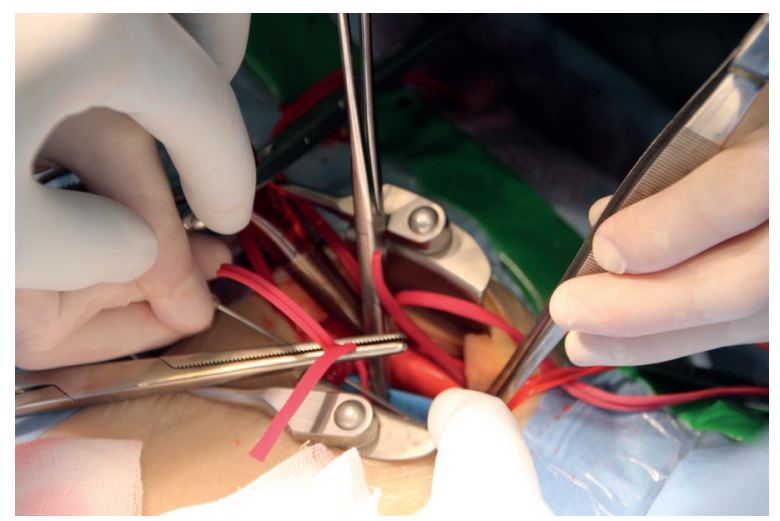

FIGURE 3. Surgical vessel preparation

high-fidelity medical simulation testing in order to prepare for real-life experience (Fig. 1, 2) [4-6].

The system of self-made silicone loops and tubing simulating blood vessels (modified polyethylene), filled with pressurized red-dyed liquid was implanted into the groin of a mannequin and covered by artificial skin. The real time scenario included all the followingcrucial steps: prehospital identification; CPR ALS; perfusion therapy (CPR-ECMO or DCD-EC$\mathrm{MO}$ ); inclusion and exclusion criteria matching; suitability for mechanical chest compression AUTOPULS (ZOLL, Chelmsford, USA); DCD confirmation and donor authorization (Fig. 3-5). The warm ischemia time, i.e. time from the first contact of mannequin to the cannulation of artificial vessels and starting in situ organ perfusion using CARDIOHELP (Maquet, Rastatt, Germany), including (PR, did not exceed two hours. In our case of a DCD-ECMO procedure, performed for the first time in Poland, its success is reassuring and helps to fight obstacles and establish a functioning programme. Soon after a successful advanced simulation test, four DCD-ECMO procedures I Maastricht category II (unsuccessful resuscitation) were performed resulting in two double successful kidney transplantations for four recipients.

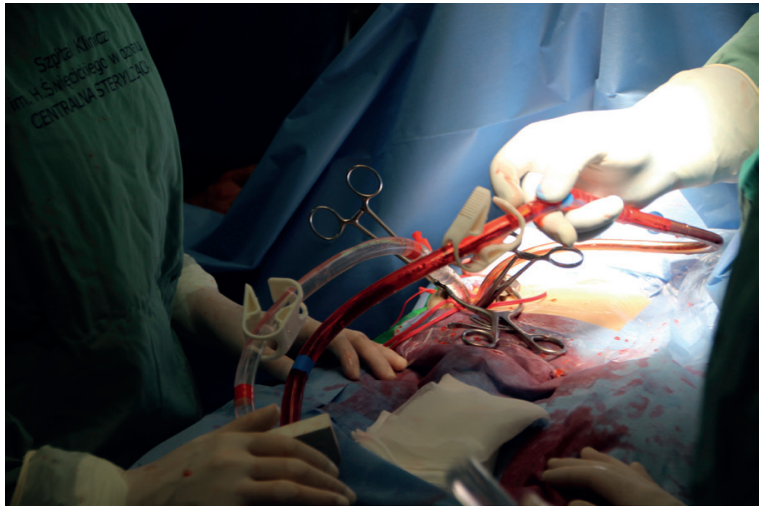

FIGURE 4. Cannulation and ECMO run

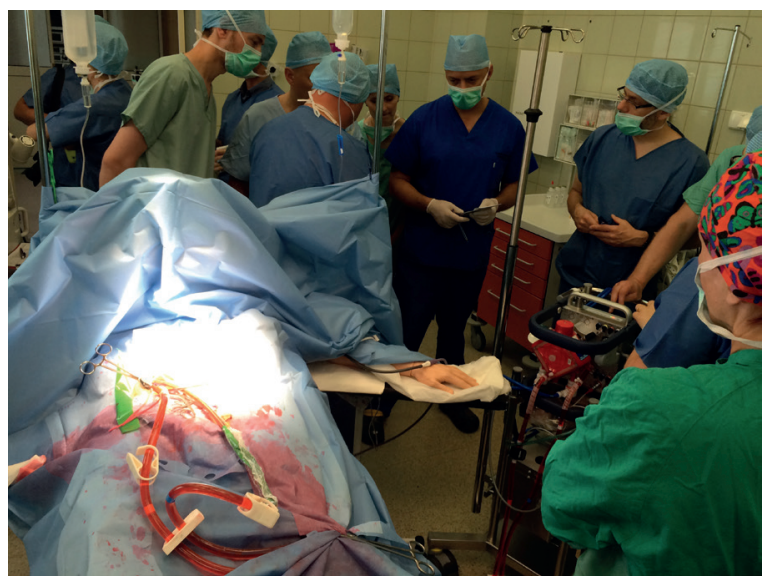

FIGURE 5. Extracorporeal support for organ donation after cardiac death simulation - $\mathrm{nECMO}$ organ perfusion

In conclusion, during debriefing, it was found that the previous training allowed one to build a procedural chain, eliminate errors at the stage of identification, notification, transportation, qualifications and ECMO organs perfusion. The training program resulted in a team appropriately qualified to successfully undertake this complex procedure. Moreover, one part of the "ECMO for Wielkopolska" program is the learning website: www.ecmo.pl.

Conflict of interest: None declared.

\section{REFERENCES}

1. Lee $\mathrm{JH}$, Hong SY, Oh CK, et al. Kidney transplantation from a donor following cardiac death supported with extracorporeal membrane oxygenation. J Korean Med Sci. 2012; 27(2): 115-119, doi: 10.3346/ jkms.2012.27.2.115, indexed in Pubmed: 22323856.

2. Ortega-Deballon I, Hornby L, Shemie SD. Protocols for uncontrolled donation after circulatory death: a systematic review of international guidelines, practices and transplant outcomes. Crit Care. 2015; 19: 268, doi: 10.1186/s13054-015-0985-7, indexed in Pubmed: 26104293. 
3. Gravel MT, Arenas JD, Chenault R, et al. Kidney transplantation from organ donors following cardiopulmonary death using extracorporeal membrane oxygenation support. Ann Transplant. 2004; 9(1): 57-58, indexed in Pubmed: 15478893.

4. Brum R, Rajani R, Gelandt E, et al. Simulation training for extracorporeal membrane oxygenation. Ann Card Anaesth. 2015; 18(2): 185-190, doi: 10.4103/0971-9784.154472, indexed in Pubmed: 25849687.
5. Burton KS, Pendergrass TL, Byczkowski TL, et al. Impact of simulation-based extracorporeal membrane oxygenation training in the simulation laboratory and clinical environment. Simul Healthc. 2011; 6(5): 284-291, doi: 10.1097/SIH.0b013e31821dfcea, indexed in Pubmed: 21642905.

6. Fehr JJ, Shepard M, McBride ME, et al. Simulation-Based Assessment of ECMO Clinical Specialists. Simul Healthc. 2016; 11(3): 194-199, doi: 10.1097/SIH.0000000000000153, indexed in Pubmed: 27093507. 